\title{
Symmetry breaking and the random-phase approximation in small quantum dots
}

\author{
Llorenç Serra, ${ }^{1}$ Rashid G. Nazmitdinov, ${ }^{1,2}$ and Antonio Puente ${ }^{1}$ \\ ${ }^{1}$ Departament de Física, Universitat de les Illes Balears, E-07122 Palma de Mallorca, Spain \\ ${ }^{2}$ Bogoliubov Laboratory of Theoretical Physics, Joint Institute for Nuclear Research, 141980 Dubna, Russia
}

(Dated: April 10, 2003)

\begin{abstract}
The random-phase approximation has been used to compute the properties of parabolic twodimensional quantum dots beyond the mean-field approximation. Special emphasis is put on the ground state correlation energy, the symmetry restoration and the role of the spurious modes within the random-phase approximation. A systematics with the Coulombic interaction strength is presented for the 2-electron dot, while for the 6- and 12-electron dots selected cases are discussed. The validity of the random-phase approximation is assessed by comparison with available exact results.
\end{abstract}

PACS numbers: 73.21.La, 73.21.-b

\section{INTRODUCTION}

The random-phase approximation (RPA) is a very successful many-body theory, widely used to describe Fermi systems in general. It was originally proposed by Bohm and Pines to describe the response function of the electron gas and, since then, it has been rederived in many different ways and contexts [1]. Important developments of this approximation for finite systems, including the establishment of a formal scheme for both excited and ground states, were obtained many years ago in nuclear physics 2]. Unfortunately, great technical difficulties have hindered for a long time the solution of the general RPA equations, imposing the recourse to severe simplifying assumptions. In recent times, however, a renewed interest in the RPA as a systematic theory of ground state correlations in atomic nuclei has appeared (see for instance Ref. 3).

In the electron-gas theory the RPA has played a benchmark role in the development of accurate exchangecorrelation energy functionals, specially in the high density limit [4]. When turning to finite electronic systems, RPA calculations are generally more involved, due to the lack of translational symmetry. Nevertheless, extensive applications to metallic clusters (see, e.g., the review by Brack [5|) and recent studies of molecules have been performed $[6]$.

A convenient starting point to treat Fermion systems is, in many cases, a mean field description like the Hartree-Fock (HF) approach. Selfconsistency between the mean field and the single-particle orbitals and total energy minimization are the basic conditions at this level. It may happen that the selfconsistent solution breaks one of the symmetries of the exact Hamiltonian, a well known phenomenon called spontaneous symmetry breaking. It has been predicted that the RPA should improve the ground state energy. More importantly, it should restore symmetries which could be broken at the mean field level. It is our aim in this work to show with a realistic calculation for interacting electrons that this is indeed the case. We have chosen as an appropriate system a two-dimensional parabolic quantum dot, partly because of the interest attracted by semiconductor nanostructures as a paradigm of artificial quantum systems, and, also, because these systems are believed to be good candidates where electron localization or formation of Wigner molecules should be relevant in some regimes (see Ref. 7 for a recent review). We remind that Wigner molecules in quantum dots are associated with the breaking of rotational symmetry of the mean field.

The RPA is, in principle, formulated to address the excited vibrational states, or vibrons for short, of a Fermion system by means of a superposition of particle-hole and hole-particle transitions on an a priori unknown correlated ground state. A basic assumption consists in treating each particle-hole pair as a boson, i.e., the so-called quasi-boson approximation [2, 8, 9]. Thus defined, the RPA spectrum describes the intrinsic vibrations of the system and, imposing the condition of excitation vacuum, also yields an approximation for the correlated ground state. In cases of mean field broken symmetry a remarkable property of the RPA is that it separates out the collective excitation associated with each broken symmetry as a zero-energy or spurious RPA mode, with an inertial parameter which is also fixed by the approximation. As we shall show below, these spurious RPA modes play a central role in the restoration of symmetries broken at the mean field level. The physical excitations of the system consist, therefore, of vibrons and collective modes associated with spurious solutions, both kinds being described within RPA.

It should be noted that the restoration of broken symmetries can also be attained via projection techniques (see Refs. [8, 9]). Examples demonstrating their use for the case of quantum dots have been recently presented by Yannouleas and Landman [10]. In this paper, however, we shall focuss our analysis on the RPA description of the ground state and its connection to the excited states.

The electronic islands formed at the GaAs-AlGaAs interface by using appropriate electrodes constitute a remarkable example of controllable quantum system. Many properties can be described, in a first approach, by using a model of electrons confined by an external potential well of parabolic type and restricting the electronic motion to the interface plane. These model quantum dots 
have been intensively studied using a variety of methods, including semiclassical, mean field and density-functional approaches as well as exact methods attempting a direct solution of the Schrödinger equation [7]. Within the latter category we find the diagonalization in a basis, the coordinate-space and the Monte-Carlo solutions. In the exact methods one normally imposes from the beginning the Hamiltonian symmetries to the solution and, therefore, the symmetry breaking is not possible (unless degeneracy is present). This striking difference with the mean-field solution does not necessarily imply that the latter one is an artifact. Indeed, the RPA provides a consistent scheme for the corresponding physical interpretation. We attempt in this work a clarification of this issue by comparing in a test case, such as small quantum dots, exact, mean-field and RPA results.

The paper is organized as follows: Section II briefly introduces the HF mean field approach and discusses the appearance of symmetry-broken solutions. Section III is devoted to the RPA excitations, introducing the quasiboson approximation and discussing the RPA spurious mode connected with a global rotation when the electrons are localized in 2-, 6- and 12-electron dots. In Sec. IV we discuss the RPA ground state focussing on the correlation energy and symmetry restoration, comparing the RPA results with exact values. The conclusions are finally drawn in Sec. V.

\section{THE MEAN-FIELD SOLUTION}

We consider a system of $N$ electrons whose motion is restricted to the $x y$ plane, where a parabolic potential induces the formation of an electron island. The full Hamiltonian reads

$$
\mathcal{H}=\sum_{i=1}^{N}\left[\frac{\mathbf{p}^{2}}{2 m}+\frac{1}{2} m \omega_{0}^{2} r^{2}\right]_{i}+\sum_{i>j=1}^{N} \frac{e^{2}}{\kappa r_{i j}},
$$

where $\kappa$ and $m$ are the dielectric constant and electron's effective mass; $\omega_{0}$ is the external confinement frequency and we have used polar coordinates $\left(r^{2}=x^{2}+y^{2}\right)$. By introducing the length $\ell_{0}=\left(\hbar / m \omega_{0}\right)^{1 / 2}$ and energy $E_{0}=$ $\hbar^{2} / m \ell_{0}^{2}=\hbar \omega_{0}$ units we may rewrite Hamiltonian (1) in terms of a single adimensional parameter

$$
R_{W}=\frac{e^{2} /\left(\kappa \ell_{0}\right)}{\hbar \omega_{0}}
$$

as

$$
\mathcal{H}=\sum_{i=1}^{N}\left[-\frac{1}{2} \nabla^{2}+\frac{1}{2} r^{2}\right]_{i}+R_{W} \sum_{i>j=1}^{N} \frac{1}{r_{i j}} .
$$

The $R_{W}$ parameter was introduced in Ref. 11 as a measure of the relative importance of electron-electron interaction to confinement potential strength. In Ref. 12 it was used as a measure to define the different phases in quantum dots and, in particular, a formation of Wigner molecules. In our unit system it is also fulfilled that $\hbar=m=1$.

\section{A. The Hartree-Fock equations}

Introducing the basis of oscillator and spin states that diagonalizes the first term in Eq. (3), $\{|a \eta\rangle ; a=$ $1, \ldots \mathcal{N} ; \eta=\uparrow, \downarrow\}$, where $a$ labels the orbital part and $\eta$ the spin, an arbitrary single-particle orbital $|i\rangle$ is expanded as

$$
|i\rangle=\sum_{a \eta} B_{a \eta}^{(i)}|a \eta\rangle .
$$

The oscillator states $|a\rangle$ are characterized by radial $\left(n_{a}\right)$ and angular momentum $\left(m_{a}\right)$ quantum numbers -see, e.g., Ref. 13. We shall assume that each orbital $i$ has non zero components only for a given spin orientation $\eta_{i}=\uparrow$ or $\eta_{i}=\downarrow$, i.e., $B_{a \eta}^{(i)}=\delta_{\eta \eta_{i}} B_{a \eta_{i}}^{(i)}$. This condition imposes good spin symmetry on the single-particle orbitals and it is the only symmetry that we shall keep, leaving totally unspecified the remaining spatial symmetries. Note that the Slater determinant built with these single-particle orbitals will be an eigenstate of the total $S_{z}$ operator but not, in general, of $\mathbf{S}^{2}$ 14]. In the chosen basis, the HF equations are written as a system of nonlinear eigenvalue equations for the matrix of $B$ coefficients. The corresponding equation for the $a$-th component of orbital $i$ reads

$$
\begin{aligned}
\varepsilon_{a}^{(0)} B_{a \eta_{i}}^{(i)}+ & \sum_{c=1}^{\mathcal{N}} B_{c \eta_{i}}^{(i)}\left[\sum_{b d=1}^{\mathcal{N}} V_{a b c d}\left(\sum_{k=1}^{N} B_{b \eta_{k}}^{(k)} B_{d \eta_{k}}^{(k)}\right)\right. \\
& \left.-\sum_{b d=1}^{\mathcal{N}} V_{a b d c}\left(\sum_{k=1}^{N} \delta_{\eta_{i} \eta_{k}} B_{b \eta_{k}}^{(k)} B_{d \eta_{k}}^{(k)}\right)\right] \\
= & \varepsilon_{i} B_{a \eta_{i}}^{(i)} .
\end{aligned}
$$

In these equations $\varepsilon_{a}^{(0)}$ is the energy of the oscillator state $|a\rangle$ and, analogously, $V_{a b c d}$ and $V_{a b d c}$ are the matrix elements of the two-body interaction defined above $\left(R_{W} / r_{12}\right)$ with the corresponding oscillator states. We can take advantage of the fact that these matrix elements can be obtained analytically in terms of the radial $\left(n_{a}\right)$ and angular momentum $\left(m_{a}\right)$ quantum numbers for each basis state $|a\rangle$.

The first and second terms within the square brackets of Eq. (5) are the well-known direct and exchange contributions to the HF problem, respectively [15]. We note that the direct term is independent of spin, while the exchange yields a different contribution for $\eta_{i}=\uparrow$ and $\eta_{i}=\downarrow$. Using the selfconsistency approach, both direct and exchange terms are determined from the 'preceding' iteration and then the eigenvalue problem can be separately solved for each spin subset. Successive repetitions of the process lead eventually to the converged solution. 
It is worth to stress that this formulation of the HF equations is particularly well suited to numerical treatment, since the use of analytical expressions 16 for $V_{a b c d}$ minimizes numerical errors. The only remaining source of numerical error is, in fact, the precission of the eigenvalue solver. Nevertheless, a necessary approximation of the method is the truncation of the basis size $\mathcal{N}$ and one must check the stability of the results with this parameter.

\section{B. Some HF results}

The oscillator basis is defined by including all states below a given cut-off $E_{c}$, i.e., $\varepsilon_{a}^{(0)}<E_{c}$. The value of $E_{c}$ has been chosen large enough to satisfy the above mentioned convergence criterion. In particular, for $N=$ 2 and 6 it sufficed to include the first 55 and 153 oscillator states, respectively. For $N=12$ we used up to 190 states which gave good HF convergence. With the purpose to present in the following sections a systematic comparison of HF, RPA and exact results with varying $R_{W}$ we have taken as test case the $N=2$ quantum dot computing its properties for $R_{W}=0.5$ to 2.5 in steps of 0.5 . Besides, some selected cases for $N=6$ and 12 have also been calculated.

The HF density

$$
\rho_{\mathrm{HF}}(\mathbf{r})=\sum_{i, o c c}\left|\varphi_{i}(\mathbf{r})\right|^{2},
$$

where $\varphi_{i}(\mathbf{r}) \equiv\langle\mathbf{r} \mid i\rangle$ refers to the HF wave functions (4), are shown in Fig. 1 for the $N=2$ system. We note that between $R_{W}=1$ and 1.5 the system changes from a circularly symmetric solution to a spontaneously broken one, where the two electrons localize in opposite positions in the mean-field frame. These intrinsic localization becomes more and more conspicuous as the $R_{W}$ parameter is increased; an evident manifestation that if electron repulsion is strong enough the favoured solution consists of particles located as far as possible from each other.

In Fig. 2 we display the density for the $N=6$ and 12 quantum dots, using $R_{W}=1.89$. This value has been chosen after Pederiva et al. [17] who, in turn, adjusted it to the experiments by Tarucha et al. 18. Both cases show a clear symmetry breaking; the $N=6$ having the electrons localized on a ring while the 12-electron dot has a central electron dimer surrounded by a ring with 10 electrons. Regarding the HF total energies, we leave the discussion for the following sections where it will be compared with RPA and exact results.

\section{RPA EXCITATIONS}

This section presents a brief discussion of the main points of the RPA for excitations, which are an essential ingredient for the ground state description in the same theory.

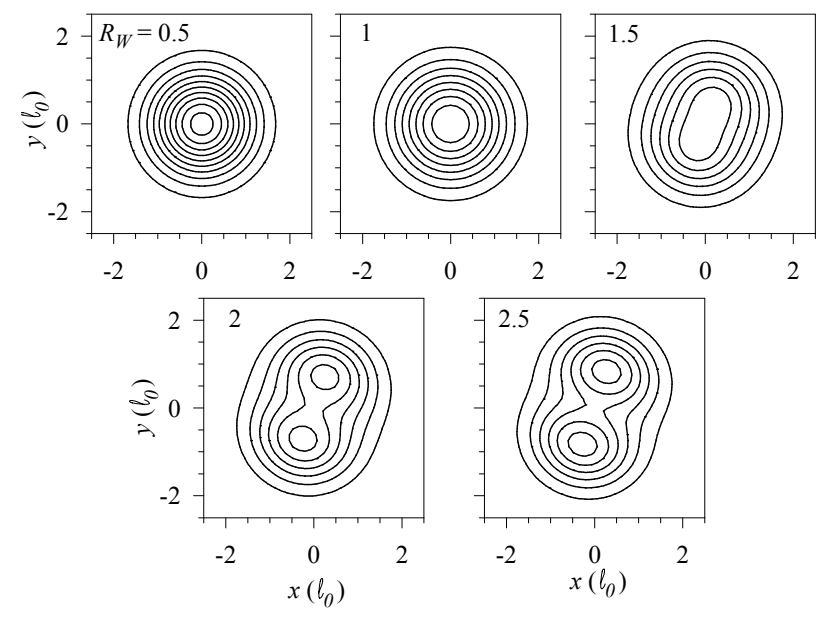

FIG. 1: HF densities for the $N=2$ quantum dot with varying $R_{W}$ parameter (shown in the upper left corner of each panel). From the outermost contour line inwards each line corresponds, respectively, to a density of $0.05,0.10,0.15 \ldots$, etc in units of $\ell_{0}^{-2}$. The cutoff in the basis has been chosen $E_{c} \approx 10.6 E_{0}$, corresponding to a basis size $\mathcal{N}=55$.

FIG. 2: Same as Fig. 1 for the $N=6$ and 12 quantum dots. For clarity the upper plots display a $3 \mathrm{~d}$ view of the corresponding densities. The contour lines are defined as in Fig. 2 and we have used $R_{W}=1.89$. The basis cutoffs for $N=6$ and 12 are $E_{c}=16 E_{0}$ and $18 E_{0}$, respectively.

\section{A. The Quasi-Boson approximation}

It is assumed that the system's excitations are created by operators of the type

$$
O_{\lambda}^{\dagger}=\sum_{m i}\left(X_{m i}^{(\lambda)} a_{m}^{\dagger} a_{i}-Y_{m i}^{(\lambda)} a_{i}^{\dagger} a_{m}\right)
$$

whose action on the as yet unknown ground state $|0\rangle$ yields the excited vibrational states $|\lambda\rangle(\lambda>0)$; i.e.,

$$
|\lambda\rangle=O_{\lambda}^{\dagger}|0\rangle \text {. }
$$

Hereafter, we shall refer to the electronic vibrational states as vibrons. Using the standard notation we refer to particle states (unoccupied HF orbitals) by subscripts $m, n$ and to hole states (below the HF Fermi level) by $i, j$. We shall denote the HF Slater determinant for $N$ electrons as $|v\rangle$ since it will act as our particle-hole vacuum. The coefficients $X^{(\lambda)}$ and $Y^{(\lambda)}$ in Eq. (7) are a set of complex amplitudes characterizing each particular excitation.

The quasi-boson approximation (QBA) amounts to treat each particle-hole pair $(m i)$ as an elementary boson, thus fulfilling

$$
\left[a_{i}^{\dagger} a_{m}, a_{n}^{\dagger} a_{j}\right] \approx\left[b_{m i}, b_{n j}^{\dagger}\right]=\delta_{m n} \delta_{i j}
$$

where the first equality introduces the boson operators $b_{m i}^{\dagger}=a_{m}^{\dagger} a_{i}$. The result (9) is exact in the average, i.e., 
when considered as expectation value with the uncorrelated vacuum $|v\rangle$ as $\left\langle v\left|\left[a_{i}^{\dagger} a_{m}, a_{n}^{\dagger} a_{j}\right]\right| v\right\rangle=\delta_{m n} \delta_{i j}$; see Refs. 2, 8, 9. In this approximation, the creation $O_{\lambda}^{\dagger}$ and annihilation $O_{\lambda}$ operators obey bosonic commutation relations

$$
\left[O_{\lambda}, O_{\lambda^{\prime}}^{\dagger}\right]=\delta_{\lambda \lambda^{\prime}}
$$

At the RPA level the eigenstates and eigenvalues are obtained by simplifying the exact equations of motion

$$
\left[H, O_{\lambda}^{\dagger}\right]=\hbar \omega_{\lambda} O_{\lambda}^{\dagger}
$$

for the vibron operators $O_{\lambda}^{\dagger}$ and excitation energies $\hbar \omega_{\lambda}$ within the particle-hole space. With the aid of the QBA the particle-hole part of the commutator $\left[H, O_{\lambda}^{\dagger}\right]$ can be derived [2, 8, 9] and Eq. (11) then transforms to a generalized eigenvalue problem, well known in the literature. We stress that henceforth all commutators are to be understood in the above QBA. The RPA equations read

$$
\left(\begin{array}{ll}
A & B \\
B^{*} & A^{*}
\end{array}\right)\left(\begin{array}{l}
X \\
Y
\end{array}\right)=\hbar \omega_{\lambda}\left(\begin{array}{r}
X \\
-Y
\end{array}\right)
$$

where the submatrices $A$ and $B$ are given by

$$
\begin{aligned}
& A_{m i n j}=\varepsilon_{m i} \delta_{m n} \delta_{i j}+V_{m j i n}-V_{m j n i} \\
& B_{m i n j}=V_{m n i j}-V_{m n j i} .
\end{aligned}
$$

Here, $\varepsilon_{m i}$ is the energy of each bosonic pair, given in terms of the HF eigenvalues as $\varepsilon_{m i}=\varepsilon_{m}-\varepsilon_{i}$.

The matrix elements in Eqs. (13) may be computed in the oscillator basis using expressions similar to those of the HF eigenvalue problem. For instance,

$$
\begin{aligned}
V_{m j i n}= & \delta_{\eta_{m} \eta_{i}} \delta_{\eta_{n} \eta_{j}} \times \\
& \sum_{a b c d=1}^{\mathcal{N}} V_{a b c d} B_{a \eta_{m}}^{(m) *} B_{b \eta_{j}}^{(j) *} B_{c \eta_{i}}^{(i)} B_{d \eta_{n}}^{(n)} .
\end{aligned}
$$

In practice, the numerical solution of Eq. (12) requires the diagonalization of a rather large matrix, whose dimension depends on the number of hole $N_{h}$ and particle $N_{p}$ states. To be consistent with our imposed spin symmetry we restrict to bosonic pairs where each member of the pair has the same spin.

\section{B. Spurious modes}

It was proved by Thouless [19] that, when the HF solution corresponds to a minimum in the energy surface, the RPA Eq. (12) provides only real frequencies $\omega_{\lambda}$. Furthemore, if the full Hamiltonian has any symmetry which is broken by the mean field there is a corresponding RPA mode with zero frequency (a spurious mode) which is orthogonal to the other vibrational (nonzero energy) modes. In other words, the generators of symmetries broken on the mean field level create eigenstates with zero energy in RPA.

As shown below, we shall be concerned with rotational symmetry for which the infinitesimal generator is the orbital angular momentum $L_{z}$. To treat the spurious mode related to rotation it is convenient to introduce the canonical conjugate operators $L_{z}$ and an angle operator $\Phi$; the latter defined by the following relations [19, 20]

$$
\begin{aligned}
& {\left[H, L_{z}\right]=0,} \\
& {[H, C]=\hbar L_{z}} \\
& {\left[L_{z}, C\right]=\hbar \mathcal{J} .}
\end{aligned}
$$

where $C=i \mathcal{J} \Phi$ is an anti-Hermitian operator and $\mathcal{J}$ is the moment of inertia.

When the above Eqs. are implemented in the RPA they lead to the so-called Thouless-Valatin moment of inertia 21] $\mathcal{J}_{0}$ and to the two RPA vectors

$$
\begin{aligned}
L_{z} & \equiv \sum_{m i}\left(\ell_{m i}^{(z)} b_{m i}^{\dagger}+\ell_{m i}^{(z) *} b_{m i}\right), \\
C & \equiv \sum_{m i}\left(c_{m i} b_{m i}^{\dagger}-c_{m i}^{*} b_{m i}\right) .
\end{aligned}
$$

The coefficients of the RPA $L_{z}$ operator are directly given by the single-particle HF matrix elements. In contrast, for the $C$ operator one needs to solve the linear system of equations

$$
\left(\begin{array}{ll}
A & B \\
B^{*} & A^{*}
\end{array}\right)\left(\begin{array}{c}
c \\
c^{*}
\end{array}\right)=\hbar\left(\begin{array}{c}
\ell^{(z)} \\
\ell^{(z) *}
\end{array}\right) .
$$

Once these two sets of coefficients are determined, the Thouless-Valatin moment of inertia $\mathcal{J}_{0}$ may be calculated as

$$
\mathcal{J}_{0}=\sum_{m i}\left(\ell_{m i}^{(z) *} c_{m i}+\ell_{m i}^{(z)} c_{m i}^{*}\right)
$$

The operators of Eqs. (16), together with the RPA vibrons $O_{\lambda}^{\dagger}, O_{\lambda}$, form a complete set for any operator linear in the bosons $b_{m i}^{\dagger}$ and $b_{m i}$. From the point of view of its Fermionic character this could represent an arbitrary one-particle-one-hole operator of the type

$$
F=\sum_{m i} f_{m i} b_{m i}^{\dagger}+f_{i m} b_{m i}
$$

The corresponding expansion in terms of RPA excitations (for a single spurious mode) reads

$$
\begin{aligned}
F & =\sum_{\omega_{\lambda}>0}\left(\left[O_{\lambda}, F\right] O_{\lambda}^{\dagger}+\left[F, O_{\lambda}^{\dagger}\right] O_{\lambda}\right) \\
& +\frac{1}{\mathcal{J}_{0}}\left([F, C] L_{z}+\left[L_{z}, F\right] C\right) .
\end{aligned}
$$

When applied to the boson operators themselves, the above result implies that we can expand each $b_{m i}^{\dagger}$ and $b_{m i}$ in terms of the vibrons $\left(O_{\lambda}^{\dagger}, O_{\lambda}\right)$ and spurious modes $\left(L_{z}, C\right)$. We shall return to this point in more detail in the next section. 


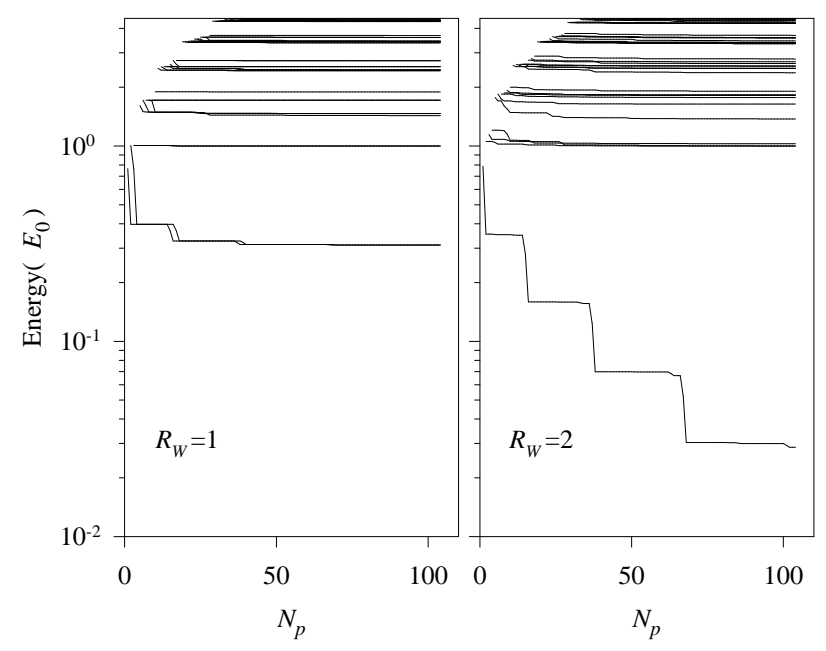

FIG. 3: Evolution of the RPA excitation spectra with the number of particle states included. The results correspond to $N=2$ with $R_{W}=1$ (left) and 2 (right). The same scale has been used in both panels for a better comparison. Note that increasingly higher RPA excitations appear as $N_{p}$ is increased. The same basis size and cutoff of Fig. 11 have been used.

\section{Strength function and sum rules}

The strength function for a general Hermitian singleparticle operator $F$ reads

$$
S(E)=\sum_{\lambda} \delta\left(E-\hbar \omega_{\lambda}\right)|\langle\lambda|F| 0\rangle|^{2} .
$$

Important quantities related to the strength function are its energy moments, usually known as sum-rules. The $k$-th moment $S_{k}$ is given by

$$
S_{k}=\sum_{\lambda}\left(\hbar \omega_{\lambda}\right)^{k}|\langle\lambda|F| 0\rangle|^{2}
$$

It is well known that the RPA fulfills several exact sum rules (see Ref. 22 for recent applications of sum rules to quantum dots). In particular, for the dipole operators $F=\sum_{i} x_{i}$ or $F=\sum_{i} y_{i}$ one has $S_{1}=\hbar^{2} N /(2 m)$. More importantly, the RPA also fulfills the Kohn theorem for parabolic confinement (see, e.g., Ref. 16) stating that the only allowed dipole excitation is the center of mass rigid motion at an energy $\hbar \omega_{0}$. These exact results constitute good checks of selfconsistency between HF and RPA, as well as of convergence with space size, in numerical calculations.

\section{Calculated spectra}

Figure 3 displays the eigenvalues $\hbar \omega_{\lambda}$ for two cases of the $N=2$ quantum dot as a function of the number of particle states included in the RPA equations. The first case $\left(R_{W}=1\right)$ is circularly symmetric at the mean field level while the second one $\left(R_{W}=2\right)$ has rotational broken symmetry (cf. Fig. 11). The evolution of the eigenvalue set with $N_{p}$ shows a remarkable difference in these two cases. While all the eigenvalues of the circular dot stabilize for a high enough value of $N_{p}$, the symmetry broken solution exhibits one state whose energy keeps decreasing as the number of particle states is increased. It clearly corresponds to the appearance of an RPA spurious mode connected with the broken rotational symmetry, which in the limit $N_{p} \rightarrow \infty$ should lie at zero energy. Though stable, the first excited state of the circular case lies rather low in energy, a hint of the proximity in $R_{W}$ value to the broken symmetry transition point. Actually, for $R_{W}=0.5$ the first vibron settles down at a higher energy, $E \approx 0.7 E_{0}$.

Figure 4 shows the corresponding spectra for the $N=$ 6 and 12 quantum dots. Both HF solutions correspond to broken symmetry cases (cf. Fig. 21) and, therefore, their RPA spectra should display spurious solutions. This is clearly the situation for $N=6$, with a well separated low energy mode. For $N=12$, however, the situation is less convincing and we actually obtain two modes which seem to separate from the higher vibron states. We stress that for the 12 electron dot the strong increase in number of particle-hole pairs prevents us from extending the calculation to higher $N_{p}$ values. Nevertheless, a tentative interpretation may be given taking into account the mean field structure of this dot shown in Fig. 2. One would expect a real spurious mode, corresponding to the rigid rotation of the full system, and a soft (low energy) mode corresponding to the rotation of the inner electron dimer with respect to the outer ring of electrons. These two modes could thus explain the behaviour of the two lower RPA eigenvalues in the right panel of Fig. 4.

We analyze next the strength function results. Figure 5 displays some representative cases, for the dot with six electrons. The upper panels show the electric $(x)$ and magnetic dipole $\left(\ell_{z}\right)$ strength functions computed within the HF method, while the lower ones are the analogous results within the RPA. In HF all excitations are above the gap between the Fermi level and the first unoccupied orbital (the HOMO-LUMO gap) which for $N=6$ takes the value $\approx 1.8 E_{0}$. In RPA, however, one obtains states below this gap. Actually, the strength function for the $\ell_{z}$ operator concentrates almost exclusively on the first RPA eigenvalue, at a very small energy; a clear manifestation of rotational spurious mode discussed above. For the

\begin{tabular}{cc|c}
$N$ & $R_{W}$ & $\mathcal{J}_{0}\left(m \ell_{0}^{2}\right)$ \\
\hline \hline 2 & 1.5 & 0.68 \\
2 & 2 & 1.26 \\
2 & 2.5 & 1.63 \\
6 & 1.89 & 16.4 \\
12 & 1.89 & 57.6 \\
\hline
\end{tabular}

TABLE I: Moments of inertia for the spurious rotational modes obtained in the Thouless-Valatin approximation (see Sec. III-B). 
FIG. 4: Same as Fig. 3 for the $N=6$ and 12 quantum dots.

FIG. 5: HF and RPA excitation cross sections for the $N=6$ quantum dot. Vertical bars show in an arbitrary scale the position and height for each state while the solid line displays the accumulated contributions to the $S 1$ sum rule. Left and right panels correspond to the dipole $(x)$ and rotation $\left(\ell_{z}\right)$ operators, respectively. The inset in the bottom-right panel shows an enlarged view, using logarithmic scales, of the RPA $\ell_{z}$ cross section.

dipole operator there is again a single mode, this time at an energy $\approx 1 E_{0}$, and the total sum rule $S 1$ is $\approx 3 E_{0} \ell_{0}^{2}$. As also discussed above, this proves that our results fulfill Kohn theorem with an excellent accuracy.

The Thouless-Valatin moments of inertia obtained for the quantum dots with spurious modes are summarized in Tab. I. These inertial parameters define physical rotational bands, characterized by the energies $\hbar^{2} M^{2} /\left(2 \mathcal{J}_{0}\right)$ with $M=0,1, \ldots 23]$. We have also checked numerically that the Thoules-Valatin moment of inertia coincides, to a good accuracy, with the value obtained from a constrained HF calculation for $\mathcal{R}=\mathcal{H}-\lambda L_{z}$ as

$$
\mathcal{J}_{0}=-\frac{d^{2}\langle\mathcal{R}\rangle}{d \lambda^{2}}=\frac{d\left\langle L_{z}\right\rangle}{d \lambda} .
$$

We stress that the equivalence between the two moments of inertia can be fulfilled if and only if a self-consistent HF minimum solution is found.

\section{THE GROUND STATE IN THE RPA}

The RPA ground state $|0\rangle$ is defined from the condition that it is the vacuum for all vibrons

$$
O_{\lambda}|0\rangle=0 .
$$

In this section we shall discuss the following aspects of the RPA ground state: i) total energy; ii) restoration of the HF broken symmetries.

\section{A. Correlation energy}

The total energy in the RPA $\left(E_{\mathrm{RPA}}\right)$ can be split in a mean field contribution $\left(E_{\mathrm{HF}}\right)$ and a correction $\left(\Delta_{\mathrm{RPA}}\right)$, as

$$
E_{\mathrm{RPA}}=E_{\mathrm{HF}}-\Delta_{\mathrm{RPA}} .
$$

Note that the correction $\Delta_{\mathrm{RPA}}$, with a minus sign, gives the standard correlation energy of the system. A decrease in ground state energy $\left(\Delta_{\mathrm{RPA}}>0\right)$ will imply an improvement with respect to the mean field theory. However, since RPA is not based on energy minimization, it is
FIG. 6: Convergence with number of particle states of the RPA correction energy $\Delta_{\text {RPA }}$. The extrapolated line as well as the exact value (horizontal line) are also shown. The exact values for $N=6$ and 12 have been taken from the Monte Carlo results of Ref. 17 .

not bound to fulfill the variational principle and, therefore, $E_{\mathrm{RPA}}$ could be even lower than the exact energy. In other words, the correlation energy could be overestimated in RPA, as it has been suggested in the literature [9].

The RPA correction reads [8, 9]

$$
\Delta_{\mathrm{RPA}}=\frac{1}{2}\left(\operatorname{Tr} A-\sum_{\omega_{\lambda}>0} \hbar \omega_{\lambda}\right) .
$$

The above Eq. (26) includes the contribution from the vibrons at positive frequencies $\omega_{\lambda}$ and, also, from the spurious mode associated with a rotational excitation. Note that Eq. (26) is the result of a partial cancellation between two large terms. In practice, this may cause $\Delta_{\text {RPA }}$ to converge rather slowly with space dimension, i.e., a high number $N_{p}$ of HF particle orbitals may be needed (see below).

\section{B. Numerical results for ground-state energies}

We have analyzed the RPA ground state for the same dots whose excited states have been discussed in the preceding sections. Figure 6 displays the evolution of the ground state energy $E_{\mathrm{RPA}}$ with number of particle states $N_{p}$. The convergence with $N_{p}$ depends on each specific case: while the $N=2, R_{W}=1$ energy converges fast enough, the $N=12$ result is clearly still increasing for the maximum $N_{p}$ used in the numerics. This is not surprising, since one naturally expects that the larger $N_{h}$ the larger the space requirements for RPA convergence. A similar conclusion may be drawn regarding the intensity of the interaction, as given by the parameter $R_{W}$. In view of the obtained results, $E_{\mathrm{RPA}}$ must be extrapolated, in general, from the range of the computationally feasible values. We have empirically found that a 4 -parameter function, $a-b /\left(1+c N_{p}\right)^{1 / d}$, leads to quite reasonable fits (see Fig. [6).

To assess the quality of $E_{\mathrm{RPA}}$ we compare next with available exact energies, i.e., with $a b$ initio solutions of the many-body Schrödinger equation. In fact, the studied cases were chosen in part to allow for this comparison. For the $N=2$ dot the exact solution can be obtained by introducing center of mass and relative coordinates. Only the relative coordinate problem needs to be solved numerically and, because of the circular symmetry, it reduces to an straightforward $1 \mathrm{~d}$ radial problem. We have solved this equation using a uniform radial grid and standard integration methods (see also Refs. 24, 26 for recent discussions of the 2-electron dot exact solution as well as 
FIG. 7: Results for the 2-electron dot as a function of $R_{W}$. Right scale corresponds to the occupation numbers of the HF hole state (solid symbols) while left scale indicates the ground state energies in HF (circles), RPA (squares) and exact solution (triangles). See the discussion about occupation numbers see in Sec. IV-C

Ref. [10] for the application of the projection methods to this system). For the larger dots we shall compare with the Monte Carlo results of Pederiva et al. 17.

Figure 7 presents a systematics for the $N=2$ dots with different values of the interaction-confinement ratio. As anticipated in Sec. IV-A, $E_{\mathrm{RPA}}$ lies below the exact value, a manifestation of an overestimated correlation energy in RPA. Nevertheless, for most of the results the RPA clearly improves the HF energy, since $E_{\mathrm{RPA}}$ is closer to the exact value than $E_{\mathrm{HF}}$. This is not the case, however, when $R_{W}=1$; the exact result lying almost halfway of RPA and HF in this instance. For $N=6$ and 12 the results in Fig. [6] increase towards the exact values. The extrapolation in these systems leads to $\Delta_{\mathrm{RPA}}(6)=1.27 E_{0}$ and $\Delta_{\mathrm{RPA}}(12)=3.21 E_{0}$; to be compared with the diffusion Monte Carlo values $\Delta_{\mathrm{MC}}(6)=1.13 E_{0}$ and $\Delta_{\mathrm{MC}}(12)=2.64 E_{0}$. The RPA overestimation of correlation energy is thus of $\approx 12 \%$ and $\approx 21 \%$ for 6 and 12 , respectively. The larger difference for the 12-electron dot could be partly attributed to the extrapolation procedure being based on a relatively small space. The deviations with respect to the exact values for $N=2$ are not surprising since RPA and HF approaches are many-body theories aiming at an accurate description of large enough systems.

\section{Occupation numbers and expectation value}

In order to construct the RPA ground state we use Eq. (24) and the condition

$$
L_{z}|0\rangle=0,
$$

that insures the rotational invariance of the ground state. One seeks solutions of the form [2, [8, 9$]$

$$
|0\rangle=N_{0} e^{S}|v\rangle
$$

with the $S$ operator involving the creation of two bosons,

$$
S=\frac{1}{2} \sum_{m i n j} Z_{m i n j} b_{m i}^{\dagger} b_{n j}^{\dagger} .
$$

In Eqs. (28) and (29) $N_{0}$ is a normalization constant and the matrix $Z_{m i n j}$ is, in general, complex and symmetric in the boson indexes, i.e., $Z_{m i n j}=Z_{n j m i}$.

Using the general indentity

$$
F e^{S}=e^{S}\left(F+[F, S]+\frac{1}{2}[[F, S], S]+\ldots\right)
$$
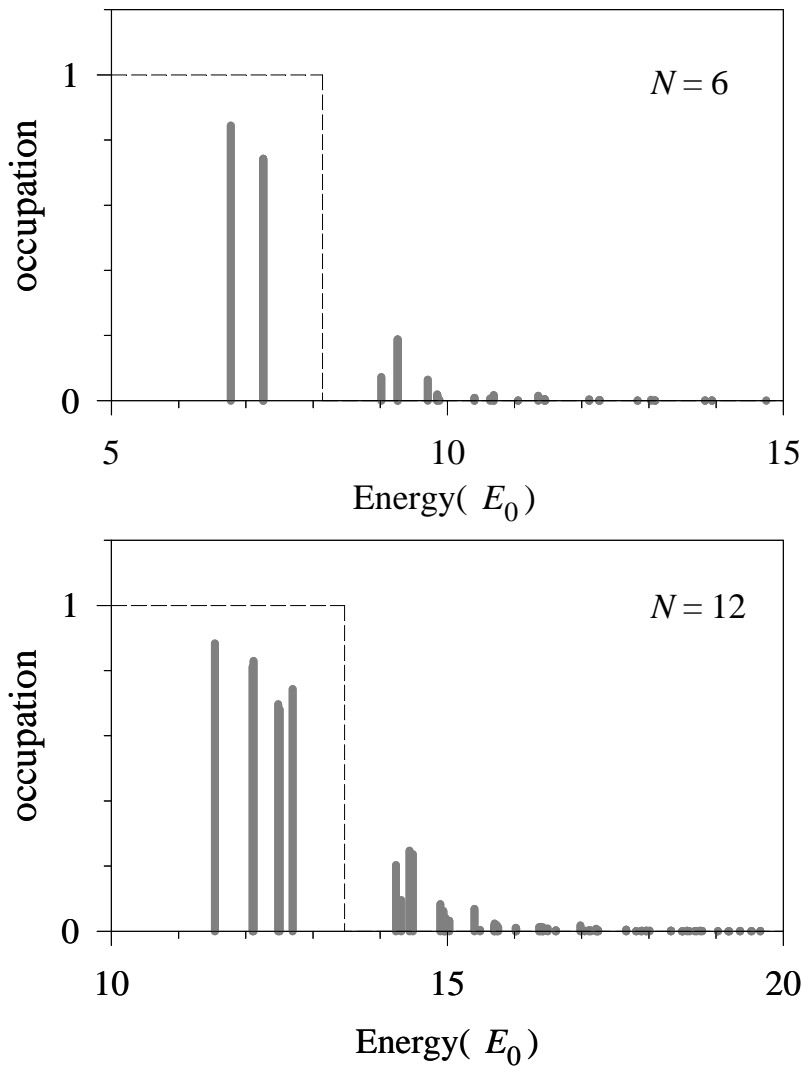

FIG. 8: Occupation numbers within RPA of the HF singleparticle orbitals. The step function shown with a dashed line separates fully occupied from unoccuppied HF orbitals in the mean field picture.

for the operators $F \equiv O_{\lambda}$ and $F \equiv L_{z}$; as well as Eq. (24), Eq. (27) and the QBA one finds

$$
(F+[F, S])|v\rangle=0 .
$$

For the case of non-broken symmetry the above requirement leads to the following equations for the $Z_{m i n j}$ coefficients

$$
Y_{m i}^{(\lambda) *}=\sum_{n j} Z_{m i n j} X_{n j}^{(\lambda) *}
$$

When the mean-field breaks rotational symmetry we have to complement the above system of equations for vibrons, which are reduced by one equation, with the additional condition for the spurious mode

$$
\ell_{m i}^{(z)}=-\sum_{n j} Z_{m i n j} \ell_{n j}^{(z) *} .
$$

Our interest is focused on the occupation numbers of the HF orbitals in the correlated ground state, $\left\langle 0\left|a_{m}^{\dagger} a_{m}\right| 0\right\rangle$ and $\left\langle 0\left|a_{i}^{\dagger} a_{i}\right| 0\right\rangle$. Using

$$
\begin{aligned}
{\left[a_{m}^{\dagger} a_{m}, b_{n j}^{\dagger}\right] } & =\delta_{m n} b_{m j}^{\dagger} \\
{\left[a_{i}^{\dagger} a_{i}, b_{n j}^{\dagger}\right] } & =\delta_{i j} b_{n i}^{\dagger}
\end{aligned}
$$


one obtains

$$
\begin{aligned}
\left\langle 0\left|a_{m}^{\dagger} a_{m}\right| 0\right\rangle & =\sum_{i n j} Z_{m i n j}\left\langle 0\left|b_{m i}^{\dagger} b_{n j}^{\dagger}\right| 0\right\rangle \\
\left\langle 0\left|a_{i}^{\dagger} a_{i}\right| 0\right\rangle & =1-\sum_{m n j} Z_{m i n j}\left\langle 0\left|b_{m i}^{\dagger} b_{n j}^{\dagger}\right| 0\right\rangle .
\end{aligned}
$$

By substituting in the above expressions the expansion of the boson operators $b_{m i}^{\dagger}$ in terms of vibrons and spurious modes, Eq. (20), we finally obtain

$$
\begin{aligned}
\left\langle 0\left|a_{m}^{\dagger} a_{m}\right| 0\right\rangle & =\frac{1}{2} \sum_{i \lambda}\left|Y_{m i}^{\lambda}\right|^{2}+\left\langle 0\left|\Phi^{2}\right| 0\right\rangle \sum_{i}\left|\ell_{m i}^{z}\right|^{2} \\
\left\langle 0\left|a_{i}^{\dagger} a_{i}\right| 0\right\rangle & =1-\frac{1}{2} \sum_{m \lambda}\left|Y_{m i}^{\lambda}\right|^{2} \\
& -\left\langle 0\left|\Phi^{2}\right| 0\right\rangle \sum_{m}\left|\ell_{m i}^{z}\right|^{2}
\end{aligned}
$$

These equations generalize the result known in the literature 2] by introducing an additional term related to the canonical variables of the spurious mode $\left\{L_{z}, \Phi\right\}$. It should be noted that the factor $1 / 2$ in Eqs. (36) and (37) is introduced following Ref. 27, where the occupation numbers were calculated using Fermionic anticommutator rules, without referring to the QBA.

The above discussion can be easily extended to obtain the expectation value of any 1-body operator such as, e.g., the particle density

$$
\hat{\rho}(\mathbf{r})=\sum_{\alpha \beta} \rho_{\alpha \beta}(\mathbf{r}) a_{\alpha}^{\dagger} a_{\beta}
$$

where indexes $\alpha$ and $\beta$ run over all the HF set of orbitals and $\rho_{\alpha \beta}(\mathbf{r})=\varphi_{\alpha}^{*}(\mathbf{r}) \varphi_{\beta}(\mathbf{r})$ (with $\varphi_{\alpha}$ the HF wave functions). Omitting for the sake of presentation the spatial dependence, one can write the RPA ground state density as

$$
\langle 0|\hat{\rho}| 0\rangle=\sum_{i} \rho_{i i}-\Delta \rho_{h}+\Delta \rho_{p}
$$

with

$$
\begin{aligned}
\Delta \rho_{h} & =\frac{1}{2} \sum_{\lambda i j m} \rho_{i j} Y_{m i}^{(\lambda) *} Y_{m j}^{(\lambda)} \\
& +\left\langle 0\left|\Phi^{2}\right| 0\right\rangle \sum_{i j m} \rho_{i j} \ell_{m j}^{(z) *} \ell_{m i}^{(z)} \\
\Delta \rho_{p} & =\frac{1}{2} \sum_{\lambda m n j} \rho_{m n} Y_{m j}^{(\lambda)} Y_{n j}^{(\lambda) *} \\
& +\left\langle 0\left|\Phi^{2}\right| 0\right\rangle \sum_{m n j} \rho_{m n} \ell_{m j}^{(z) *} \ell_{n j}^{(z)}
\end{aligned}
$$

The occupation numbers Eqs. (36) and (37), and density expectation value Eq. (39) depend on the matrix element $\left\langle 0\left|\Phi^{2}\right| 0\right\rangle$. Involving the two-body operator $\Phi^{2}$ this matrix element is not determined within the QBA
[20]. Therefore, we propose the following procedure to fix its value. Treating the unknown matrix element as a parameter $\left(\gamma \equiv\left\langle 0\left|\Phi^{2}\right| 0\right\rangle\right)$ we introduce the function

$$
F(\gamma)=\int d \mathbf{r}[\rho(\gamma, \mathbf{r})-\tilde{\rho}(\gamma, r)]^{2}
$$

where $\rho(\gamma, \mathbf{r})$ refers to the RPA density Eq. (39) and

$$
\tilde{\rho}(\gamma, r)=\frac{1}{2 \pi} \int_{0}^{2 \pi} d \theta \rho(\gamma, \mathbf{r})
$$

is the corrresponding angular average. Since $|0\rangle$ is rotationally invariant, it is natural to require the physical value $\gamma=\gamma_{0}$ to be a minimum of $F(\gamma)$. Therefore, it fulfills the condition

$$
\left.\frac{d F(\gamma)}{d \gamma}\right|_{\gamma=\gamma_{0}}=0
$$

Resolving this equation we obtain the unknown quantity as

$$
\gamma_{0}=-\frac{A}{B}
$$

where

$$
\begin{aligned}
A & =\int d \mathbf{r} \sum_{k=0}^{2} \sum_{m=1}^{2}(-1)^{k+m}\left(a_{k}-\tilde{a}_{k}\right)\left(b_{m}-\tilde{b}_{m}\right) \\
B & =\int d \mathbf{r}\left(\sum_{m=1}^{2}(-1)^{m}\left(b_{m}-\tilde{b}_{m}\right)\right)^{2}
\end{aligned}
$$

and we have defined the $a$ and $b$ coefficients according to Eqs. (40) and (41) as

$$
\begin{aligned}
\Delta \rho_{h} & \equiv a_{1}+\gamma b_{1} \\
\Delta \rho_{p} & \equiv a_{2}+\gamma b_{2} \\
a_{0} & \equiv \sum_{i} \rho_{i i} .
\end{aligned}
$$

The tilde coefficients are defined through analogous equations with the circularly averaged densities.

\section{Numerical expectation values}

The above discussed Fig. 7 contains also the numerical values of the hole state occupation for $N=2$-there is only one occuppied orbital (spin degenerate) in this case. The occupation is close to 1 for weak interaction (low $R_{W}$ ) and it decreases in an important way as the interaction strength is increased. This proofs that the RPA ground state deviates from the HF one when interaction becomes strong and correlations allow particles and holes to be found above and below the Fermi level, respectively. The occupations for the other two dots analyzed in this work are shown in Fig. 8. In these cases 


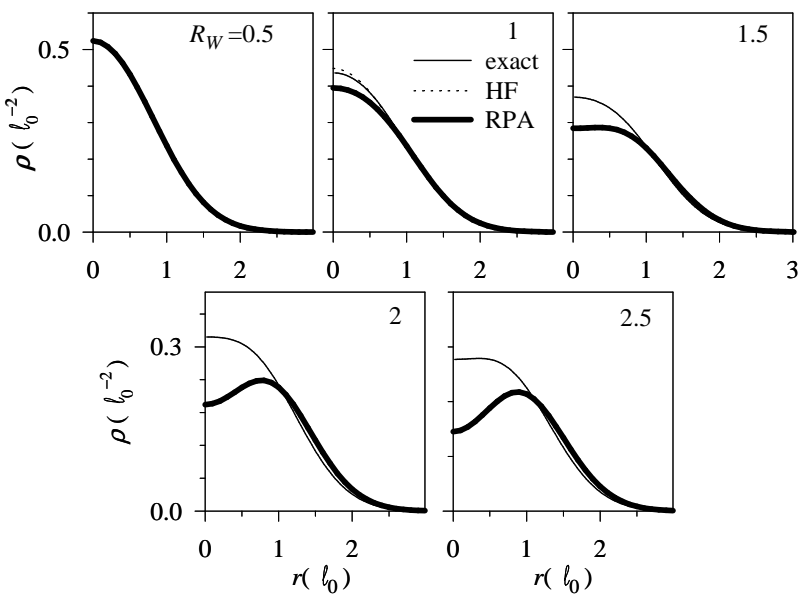

FIG. 9: Comparison of RPA and exact radial densities for the $N=2$ dot with different $R_{W}$ parameters. In the $R_{W}=0.5$ and 1 cases the HF density, which is circular, is also displayed.

we have two (for $N=6$ ) and four (for $N=12$ ) different hole levels with occupations varying with their respective energies. In general, the RPA occupation numbers show a smoother distribution than the HF ones, the maximum variations occurring nearby the Fermi level.

The RPA density is compared with the exact one in Fig. 9 for the 2-electron dot. For all $R_{W}$ parameter values the RPA density is circularly symmetric; fulfilling the symmetry restoration discussed above. Therefore, in Fig. 9 we focus exclusively on the radial dependence. While the dot edge is well reproduced, a conspicuous feature is the underestimation of the central density by the RPA, specially at large $R_{W}$ values. Our result is in qualitative agreement with that of Reinhard 28] for electrons in jellium spheres (where a circularly symmetric mean field was imposed). As shown by Reinhard the RPA correctly describes the low $q$ components (large $r$ 's) of the form factor $F(q)=\int d r \exp (i q r) \rho(r)$, but it fails for the large $q$ contributions (low $r$ 's). The overcorrection of both central density and correlation energy seem thus to be peculiarities of the RPA.

Analogous results for the 6- and 12-electron dots are presented in Fig. 10 An excellent restoration of the circular symmetry is obtained for the $N=6 \operatorname{dot}$-we remind the reader that the corresponding HF density was shown in Fig. 22 For $N=12$ the RPA density, though more circular than the HF one, still has some residual deformation. This can be surely attributed to incompleteness of the RPA space considered in the numerical calculation. In fact, for this dot the spurious mode separation (Fig. (4), the ground state energy (Fig. [6), and the density (Fig. 10) are all indicating that convergence with number of particle states is rather slow and, therefore, very difficult to be achieved in a numerical calculation. We emphasize that circular symmetry can be restored within the RPA only if the contribution from the spurious mode is taken into account.
FIG. 10: RPA symmetry restoration of the HF densities displayed in Fig. 2

\section{SUMMARY AND OUTLOOK}

The RPA is a general framework where mean field theory can be improved. Focussing on two-dimensional quantum dots we have reviewed the description of excited and ground states within the RPA. Some of the results known in the literature have been generalized to include broken symmetries at the mean field level. Quite importantly, the RPA provides a physical interpretation of this broken-symmetry phenomenon: mean-field solutions lacking one symmetry of the Hamiltonian represent, according to the RPA, true internal deformations of the system having an associated collective motion at vanishing energy (the spurious RPA mode). The ground state energy, occupation numbers, as well as general expectation values with 1-body operators, including the spuriousmode contribution, have been obtained. We proposed the procedure to restore the rotational symmetry broken at the mean field, which can be extended for other symmetry breaking cases.

Small quantum dots have provided us a good scenario to assess the above properties of the RPA by numerical calculations. For large enough values of the interactionconfinement ratio $R_{W}$ the HF mean field breaks circular symmetry; the electrons being localized in specific geometric distributions. In these cases we obtain an spurious RPA mode related to the global rotation, with its associated moment of inertia. The corresponding rotational spectra may be associated with the rotation of a Wigner molecule 29]. A systematics with $R_{W}$ for the 2-electron dot has been presented, while for 6- and 12-electron dots the calculations have been performed with an $R_{W}$ value suggested by the experiments.

When comparing with exact results, the RPA generally overestimates the correlation energy and the central dot densities by an amount which depends on the electron number and the Hamiltonian parameters. Another general behaviour of RPA is the slow convergence with space dimensions, requiring in practical applications a very high number of empty HF orbitals. For systems with many electrons this renders the numerical calculation quite a formidable task. In spite of these difficulties the RPA corrections essentially improve the HF energies with respect to exact values. It should be noted that the QBA only approximately satisfies the Pauli principle (see Eq.(9)). To solve this deficiency of the QBA could be one way to improve the description of the ground state. On the other hand, a more systematic treatment of the ground state energies would require, probably, alternative many body techniques.

The exact spin symmetry has been imposed on the single-particle states in this work, both at the HF and RPA levels. An interesting extension is, therefore, to re- 
lax this constraint allowing the HF orbitals to be general two-component spinors. The allowed symmetry breaking would eventually lead to ground states consisting of vectorial spin textures, lacking a single quantization axis. This type of states have been predicted for quantum dots in large magnetic fields and the question arises what collective spurious modes will be associated with them. This and related issues are left for a future work.

\section{Acknowledgments}

This work was supported by Grant No. BFM200203241 from DGI (Spain). R. G. N. gratefully acknowledges support from the Ramón y Cajal programme (Spain).
[1] D. Pines, The Many-Body Problem (Addison-Wesley, Reading , 1997).

[2] D. J. Rowe, Nuclear Collective Motion (Methuen, London, 1970).

[3] G.F. Bertsch and K. Hagino, Yad. Fiz. 64, 646(2001) (Phys. Part. Nucl. 64, 588 (2001)); R. G. Nazmitdinov, D. Almehed, F. Dönau, Phys. Rev. C 65, 041307(R) (2002); C. W. Johnson and I. Stetcu, Phys. Rev. C 66, 064304 (2002).

[4] D. C. Langreth and J. P. Perdew, Phys. Rev. B 15, 2884 (1977).

[5] M. Brack, Rev. Mod. Phys. 65, 677 (1993).

[6] F. Furche, Phys. Rev. B 64, 195120 (2001).

[7] S. M. Reimann and M. Manninen, Rev. Mod. Phys. 74, 1283 (2002).

[8] P. Ring and P. Schuck, The Nuclear Many-Body Problem, (Springer, New York, 1980).

[9] J. P. Blaizot and G. Ripka, Quantum Theory of Finite Systems (MIT, Cambridge, MA, 1986).

[10] C. Yannouleas and U. Landman, J. Phys.: Condens. Matter 14, L591 (2002); Phys. Rev. B 66, 115315 (2002).

[11] M. Wagner, U. Merkt, and A. V. Chaplik, Phys. Rev. B 45, 1951 (1992).

[12] C. Yannouleas and U. Landman, Phys. Rev. Lett. 82, 5325 (1999).

[13] L. Jacak, P. Hawrylak, and A. Wojs, Quantum Dots (Springer, Berlin, 1998).

[14] We shall not discuss the $\mathbf{S}^{2}$ symmetry restoration in the present work. Involving a two-body operator, this symmetry is not automatically included in the RPA. It can be dealt, however, with projection methods [10].

[15] We learned from one of the referees that this approach is known in Quantum Chemistry as the Berthier-PopleNesbet equations; see G. Berthier, Comptes Rendus de l'Academie des Sciences 238, 91 (1954); J. A. Pople and R. K. Nesbet, J. Chem. Phys. 22, 571 (1954).

[16] T. Chakraborty, Quantum Dots: A Survey of the Properties of Artificial Atoms (North-Holland, Amsterdam,
1999).

[17] F. Pederiva, C. J. Umrigar, and E. Lipparini, Phys. Rev. B 62, 8120 (2000).

[18] S. Tarucha, D.G. Austing, T. Honda, R.J. van der Hage, and L. P. Kouwenhoven, Phys. Rev. Lett. 77, 3613 (1996).

[19] D. J. Thouless, Nucl. Phys. 21, 225 (1960); ibid 22, 78 (1961).

[20] E. R. Marshalek and J. Weneser, Ann. Phys. (N.Y.) 53, 569 (1969).

[21] D. J. Thouless and J. G. Valatin, Nucl. Phys. 31, 211 (1962).

[22] E. Lipparini, N. Barberan, M. Barranco, M. Pi, and Ll. Serra, Phys. Rev. B 56, 12375 (1997); Ll. Serra, M. Barranco, A. Emperador, M. Pi, and E. Lipparini, ibid 59, 15290 (1999).

[23] Note that for small values of $\mathcal{J}_{0}$ the collective rotation with angular momentum $M$ could be higher in energy than the lowest vibron with the same angular momentum. This would imply a departure of the $M^{2}$ dependence for the lowest state of each angular momentum, i.e., of the yrast band. We believe this could explain similar deviations found in the exact calculations of Ref. 24] as well as the appearance of the so-called magic angular momenta 25]. A more detailed analysis of this effect is planned for a future work.

[24] C. Yannouleas and U. Landman, Phys. Rev. Lett. 85, 1726 (2000).

[25] W. Y. Ruan, Y. Y. Liu, C. G. Bao, and Z. Q. Zhang, Phys. Rev. B 51, 7942 (1995); P. A. Maksym, Phys. Rev. B 53, 10871 (1996).

[26] N. S. Simonović and R. G. Nazmitdinov, Phys. Rev. B 67, 041305(R) (2003).

[27] D. J. Rowe, Phys. Rev. 175, 1283 (1968).

[28] P.-G. Reinhard, Phys. Lett. A 169, 281 (1992).

[29] H.-M. Müller and S. E. Koonin, Phys. Rev. B 54, 14532 (1996). 
This figure "fig2.gif" is available in "gif" format from: http://arxiv.org/ps/cond-mat/0304246v2 
This figure "fig4.gif" is available in "gif" format from: http://arxiv.org/ps/cond-mat/0304246v2 
This figure "fig5.gif" is available in "gif" format from: http://arxiv.org/ps/cond-mat/0304246v2 
This figure "fig6.gif" is available in "gif" format from: http://arxiv.org/ps/cond-mat/0304246v2 
This figure "fig7.gif" is available in "gif" format from: http://arxiv.org/ps/cond-mat/0304246v2 
This figure "fig10.gif" is available in "gif" format from: http://arxiv.org/ps/cond-mat/0304246v2 